Mach-Zehnder Modulator performance using the Comet Laser facility and implications for use on NIF

B. Beeman, A. G. MacPhee, J. R. Kimbrough, G. A. Lacaille, M. A. Barrios, J. Emig, J. R. Hunter, E. K. Miller, W. R. Donaldson

July 12,2012

SPIE

San Diego, CA, United States

August 12, 2012 through August 16, 2012 
This document was prepared as an account of work sponsored by an agency of the United States government. Neither the United States government nor Lawrence Livermore National Security, LLC, nor any of their employees makes any warranty, expressed or implied, or assumes any legal liability or responsibility for the accuracy, completeness, or usefulness of any information, apparatus, product, or process disclosed, or represents that its use would not infringe privately owned rights. Reference herein to any specific commercial product, process, or service by trade name, trademark, manufacturer, or otherwise does not necessarily constitute or imply its endorsement, recommendation, or favoring by the United States government or Lawrence Livermore National Security, LLC. The views and opinions of authors expressed herein do not necessarily state or reflect those of the United States government or Lawrence Livermore National Security, LLC, and shall not be used for advertising or product endorsement purposes. 


\title{
Mach-Zehnder Modulator performance using the Comet Laser facility and implications for use on NIF
}

\author{
B. Beeman ${ }^{1}$, A.G. MacPhee ${ }^{1}$, J.R. Kimbrough ${ }^{1}$, G.A. Lacaille ${ }^{1}$, M.A. Barrios ${ }^{1}$, J. Emig ${ }^{1}$, \\ J.R. Hunter ${ }^{1}$, E. K. Miller ${ }^{2}$, W. R. Donaldson ${ }^{3}$ \\ ${ }^{1}$ Lawrence Livermore National Laboratory \\ ${ }^{2}$ National Security Technology \\ ${ }^{3}$ Laboratory for Laser Energetics
}

\begin{abstract}
We present performance characterization measurements for Mach-Zehnder optical modulators to be fielded on NIF as a signal path upgrade for various diagnostics. Two different operating configurations will be explored including in phase quadrature and 90deg I/Q operation. Impulse response functions of $\mathrm{x}$-ray emissions were conducted using the COMET laser facility at the Lawrence Livermore National Laboratory. Results from these short pulse laser driven plasma experiments are given along with comparisons to other recording instrumentation
\end{abstract}

\section{Introduction}

Diagnostic instruments on the National Ignition Facility (NIF) fusion laser system are required to provide high bandwidth, high fidelity measurements of physics phenomenon occurring in a high radiation and electromagnetic interference (EMI) environment. Due to the high radiation environment at NIF, recording instrumentation must be relocated to the diagnostic mezzanine, thus requiring long transmission cable runs and corresponding vulnerability to EMI pickup on the cables.. In addition there are many recording channels to which the considerations must apply. Of the two diagnostics we focus on in the paper one, DANTE, comprises two instruments, each with $18 \mathrm{X}$-ray diodes (XRDs) and is primarily concerned with temporal shape and high dynamic range. The second system, South Pole Bang Time, is used for bang time estimation and requires high signal-to-noise ratio data.

The use of Mach-Zehnder modulators addresses the above concerns by allowing data transmission via optical fibers and the use of modern high-bandwidth digitizers [REF Miller et al, 2010]. The fiber optics isolate the recording equipment from the harsh environment and potentially high signal amplitudes, thus protecting the delicate front end of the digitizers from over voltage conditions. Further, the modern digitizers offer recording durations more than 10,000 times longer than our 20ns events, facilitating time-multiplexing of multiple detector signals and multiple copies of each signal [REF Donaldson et al, 2010] onto a single digitizer channel reducing costs up front, and allowing for upgrade paths as new products hit the market.

For DANTE, multiplexing is highly desired in order to reduce digitizer costs. High dynamic range is also necessary, since signal levels will vary from $\sim 50 \mathrm{mv}$ to $200 \mathrm{v}$, depending on the experimental design of the target and laser drive. Currently, physicists calculate attenuator values for each shot, and diagnostic technicians install attenuators on the signal cables to accommodate the expected XRD signal levels within the $20 \mathrm{mV}$ to $5 \mathrm{~V}$ recording range of the existing recorders. The goal of the new EO recording system is to seamlessly cover this entire operating range with no reconfiguration required.

The South Pole Bang Time instrument uses polycrystalline diamond photoconductive detectors (PCD) to measure the $\mathrm{x}$-ray bang time. The signal of interest appears as a small inflection point on the falling edge of a much larger signal as shown in figure 1. A high signal-to-noise (SNR) is required to recover the signal of 
interest by deconvolving the impulse response function of the PCD and recording system during the data analysis.

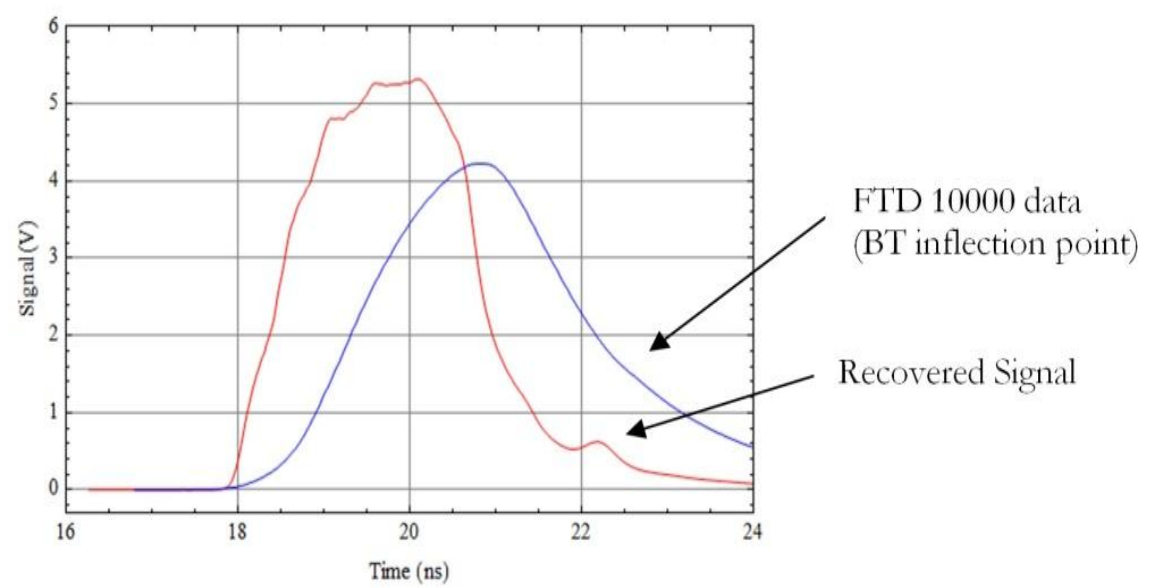

Figure 1. Raw South Pole Bang Time waveform and processed waveform to locate the bang time

\section{Experimental}

Prototype systems based upon previous work at Rochester's LLE facility were tested on the COMET laser at LLNL. COMET is capable of producing 5ps laser pulses of sufficient energy to allow the generation of Instrument Response Functions (IRF) required to recover the bang time information on the South Pole Bang Time (SPBT) diagnostic. In addition COMET's "long pulse" mode produces $\sim 2 \mathrm{~ns}$ laser pulses which allow direct comparison between a conventional DANTE recording system and the EO data acquisition system.

\subsection{SPBT Instrument Response Function}

Utilizing the short pulse (5ps) capabilities of the COMET laser the Instrument Response Function of the detection system can be obtained. Splitting the detector signal allows the simultaneous comparison of the IRF of the traditional system with long copper cables and the IRF of the Mach Zehnder system. The two systems are expected to produce different IRFs due to MachZehnder characteristics, however each should result in usable IRFs to allow recovery of the signal incident on the detector. The COMET test setup is shown in figure 2. 


\section{SPBT COMET Single Channel Config. with Mach-Zehnder Components 11/11/2011}

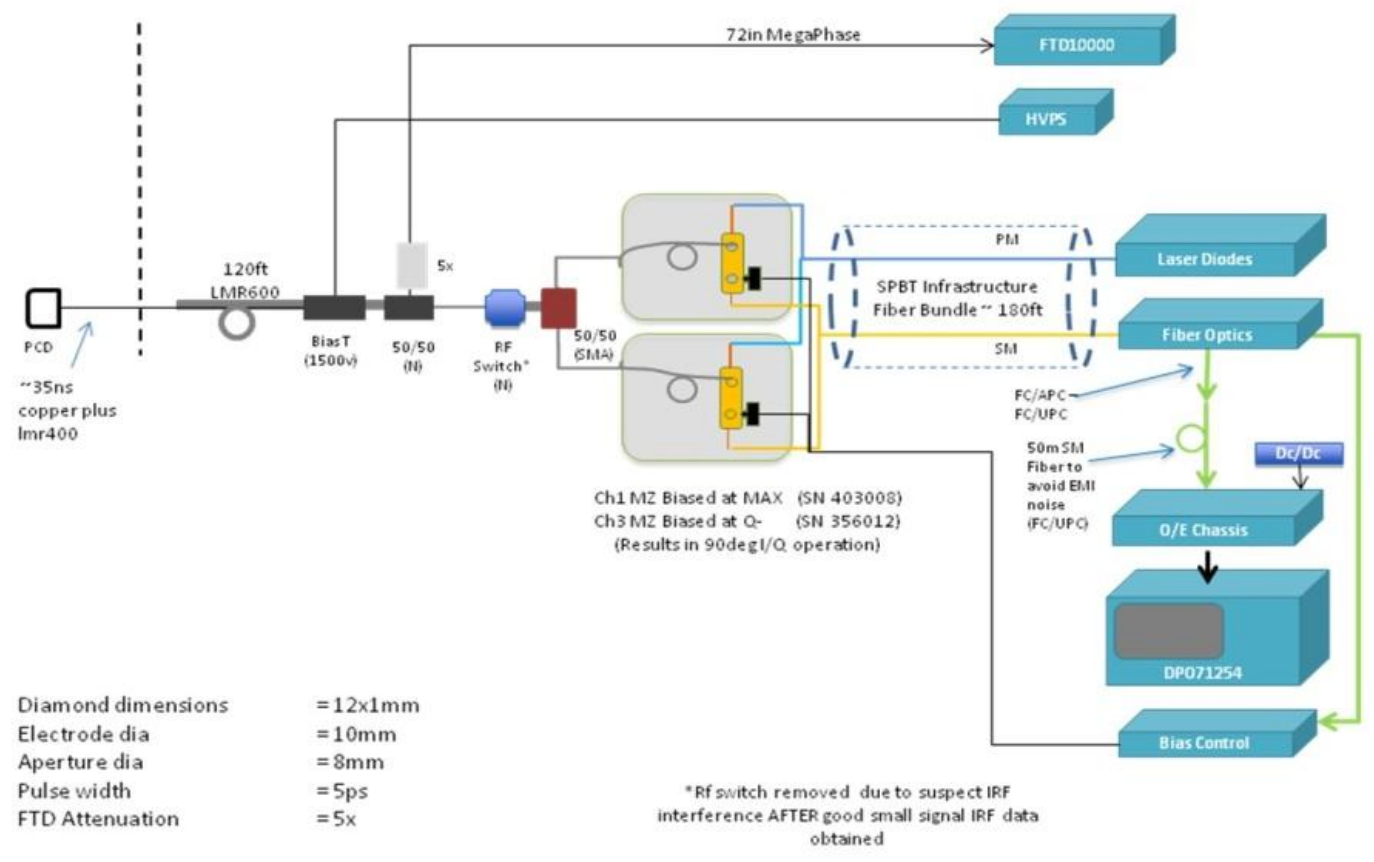

Figure 2. Comet test setup to compare Mach-Zehnder and copper cable systems.

The raw data recorded on the Tektronix DPO $71254 \mathrm{C}$ which is a $12.5 \mathrm{GHz}$ digitizer is comprised of two traces, one for each Mach-Zehnder modulator as shown in Figure 3. Post processing includes time alignment, compensation for the non-linear response of the modulators, and phase unwrapping to produce a single instrument response. Figure 4 shows the IRF for both systems.

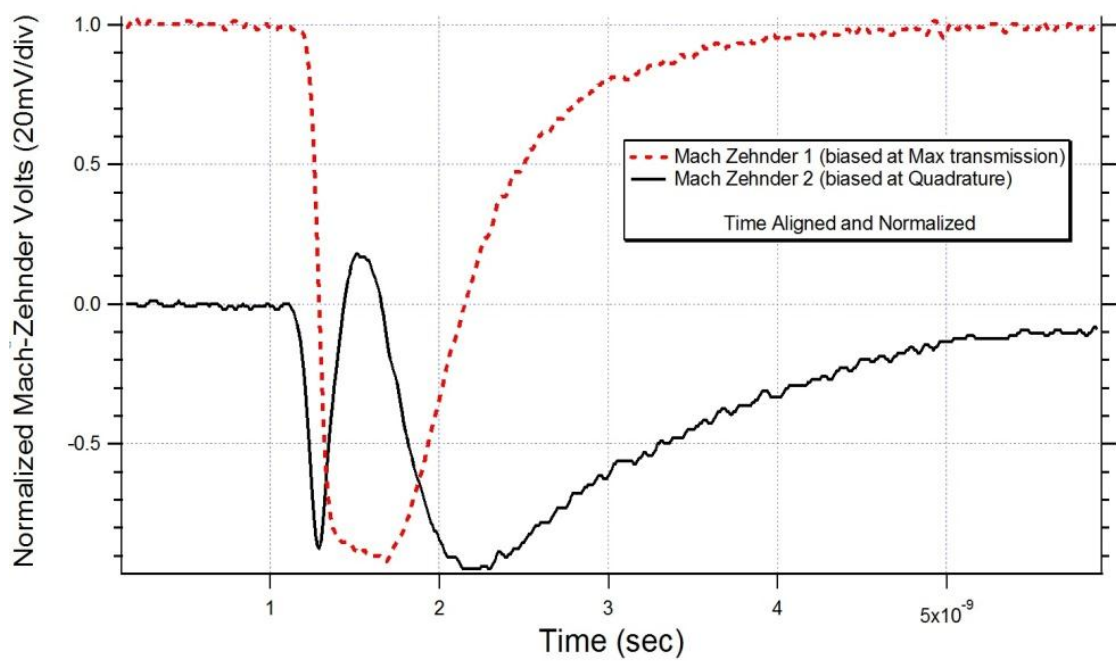

Figure 3. Mach-Zehnder waveforms from the Tektronix DPO71254 digitizer 


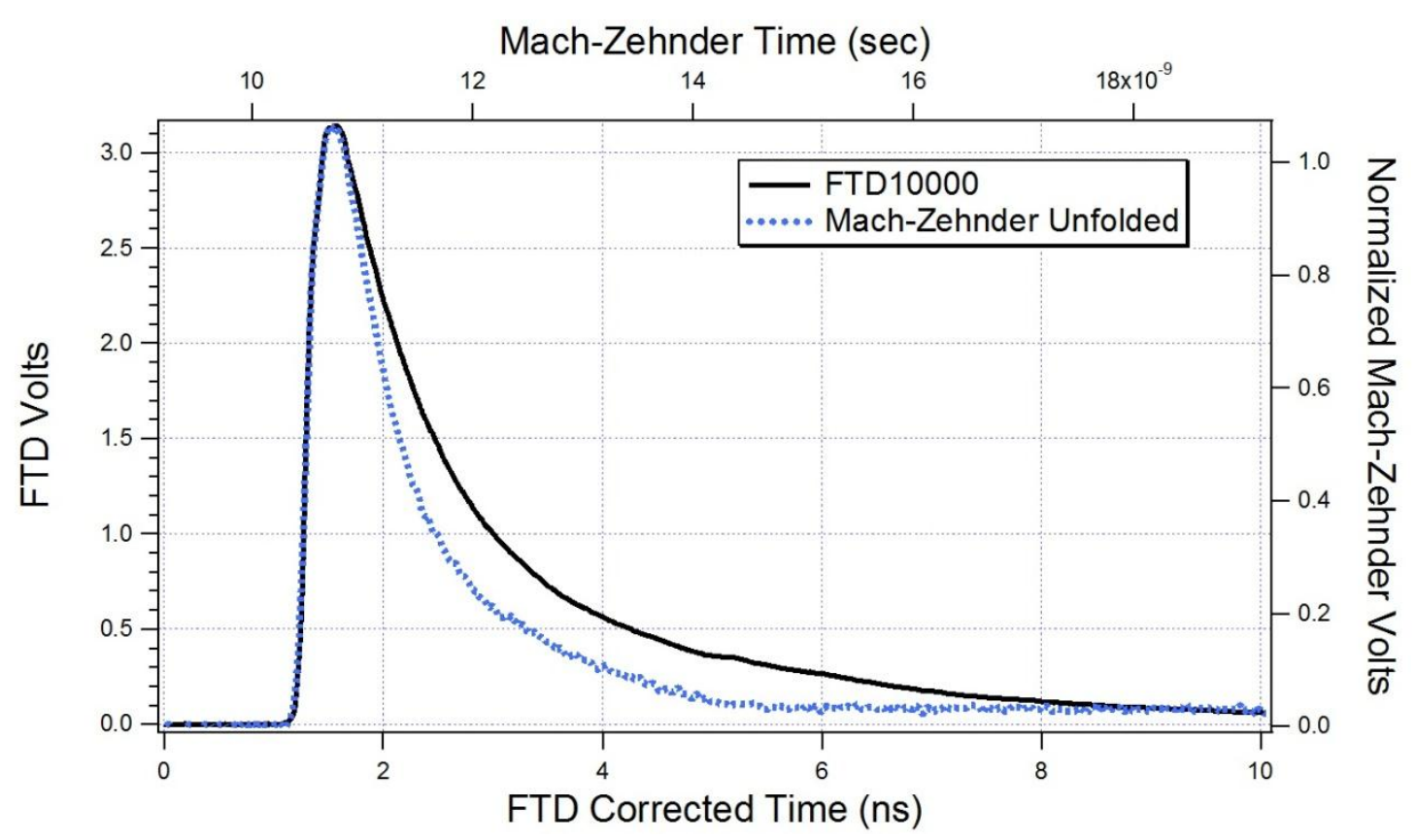

Figure 4. Instrument Response Functions of the Mach-Zehnder and the copper cable systems

The degree of deconvolution required is very sensitive to any noise recorded along with the signal and can be a major source of error in the bang time estimation. The overall noise is $4.52 \mathrm{mV}$ with the digitizer contributing $4.5 \mathrm{mV}$. In order to estimate the effects of noise upon the deconvolution process a reference 100ps FWHM Gaussian signal was generated with the peak located at $1.01 \mathrm{~ns}$. This reference signal was then convolved with the Instrument Response Function to simulate a signal as it would be recorded on the digitizer. Actual digitizer noise was added to this convolved signal and finally deconvolved to obtain an estimate of the original signal. This was repeated until the bang time estimation failed the requirement of less than 30ps resulting in a minimum signal to noise ratio of approximately $100\left(\mathrm{~V}_{\mathrm{pk}} / \mathrm{V}_{\mathrm{pk}-\mathrm{pk}}\right)$.

Table 1. Summary of SPBT Mach-Zehnder tests and simulations

\begin{tabular}{|c|c|c|c|c|c|c|c|}
\hline $\begin{array}{l}\text { Peak Volts } \\
\text { @ Mach- } \\
\text { Zehnder } \\
\text { System } \\
\text { Input }\end{array}$ & $\begin{array}{c}\text { Mach- } \\
\text { Zehnder } \\
\text { Sensitivity } \\
(\mathrm{V} \pi)\end{array}$ & $\begin{array}{c}\text { Mach } \\
\text { Zehnder } \\
\text { Model \# }\end{array}$ & $\begin{array}{c}\text { Mach } \\
\text { Zehnder } \\
\text { Serial } \\
\text { Numbers }\end{array}$ & $\begin{array}{l}\text { Measured } \\
\text { Response } \\
\text { (Rise time) }\end{array}$ & $\begin{array}{c}\text { Number } \\
\text { of Phase } \\
\text { Wraps }\end{array}$ & $\begin{array}{c}\begin{array}{c}\text { Signal to Noise } \\
\text { Ratio }\end{array} \\
\text { Peak Signal Volts } \\
\text { Peak to Peak Noise }\end{array}$ & $\begin{array}{l}\text { Expected } \\
\text { BT Error }\end{array}$ \\
\hline $15 v$ & $4.16 \mathrm{v}$ & $\begin{array}{c}\text { LN95-10- } \\
\text { S-A-A- } \\
\text { NS-VPI }\end{array}$ & $\begin{array}{l}403008 \\
356012\end{array}$ & $\sim 85 \mathrm{ps}$ & 1.3 & $>100$ & $<10 \mathrm{ps}$ \\
\hline
\end{tabular}

\section{SPBT Conclusion:}

Exploiting the phase wrapping characteristic of the Mach-Zehnders effectively increased the SNR beyond the limit of the recording digitizer. Using the IRF derived at COMET along with offline simulations, the Mach-Zehnder system can meet the specification of <30ps Bang Time Error given an input signal $>15 \mathrm{v}$, resulting in a Signal to Noise Ratio $>100$, with a Response time $<85$ ps. 


\subsection{DANTE Electro-Optic Data Acquisition System}

Accurate temporal reconstruction and high total system dynamic range are the key requirements for the DANTE EO system. Expected Dante signals vary from $50 \mathrm{mV}$ to $200 \mathrm{v}$ for a signal dynamic range of 4000:1. The recording system requirement is then $>8,000: 1\left(\mathrm{~V}_{\mathrm{pk}} / \mathrm{V}_{\mathrm{n} \_\mathrm{pk} \text {-pk }}\right)$ or $\sim 48,000: 1\left(\mathrm{~V}_{\mathrm{pk}} / \mathrm{V}_{\mathrm{n} \_\mathrm{RMS}}\right)$. Due to the large number of $\mathrm{X}$-Ray detectors, multiplexing signals onto a single digitizer channel is also required to reduce system costs.

Utilizing the same test configuration as SPBT with COMET running at $\sim 2 \mathrm{~ns}$, good agreement was obtained between the traditional recording system and the EO system.

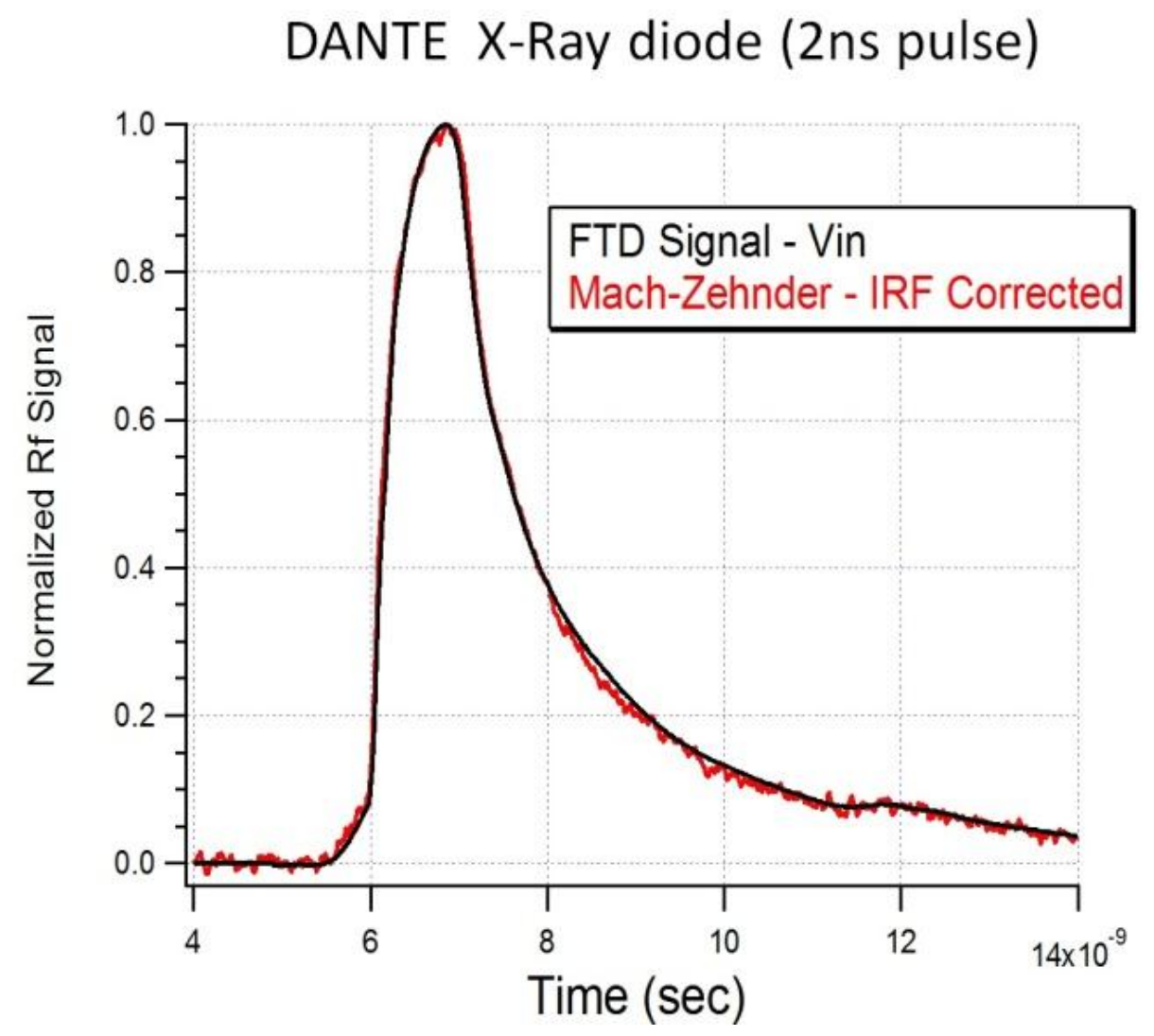

Figure 5. Conventional and EO Dante 2ns Shot Data 
Testing has also progressed well on a full DANTE EO system capable of multiplexing 6 Mach-Zehnders while supporting 2 XRDs on a single digitizer channel. Primary goals are the demonstration of $\sim 50 \mathrm{mV}$ signal detection as well as the multiplexing capability.

\section{DANTE MZ Single Lambda Multiplexing}

- 2ea XRDs per scope input

- Use other replicator input for 2nd XRD

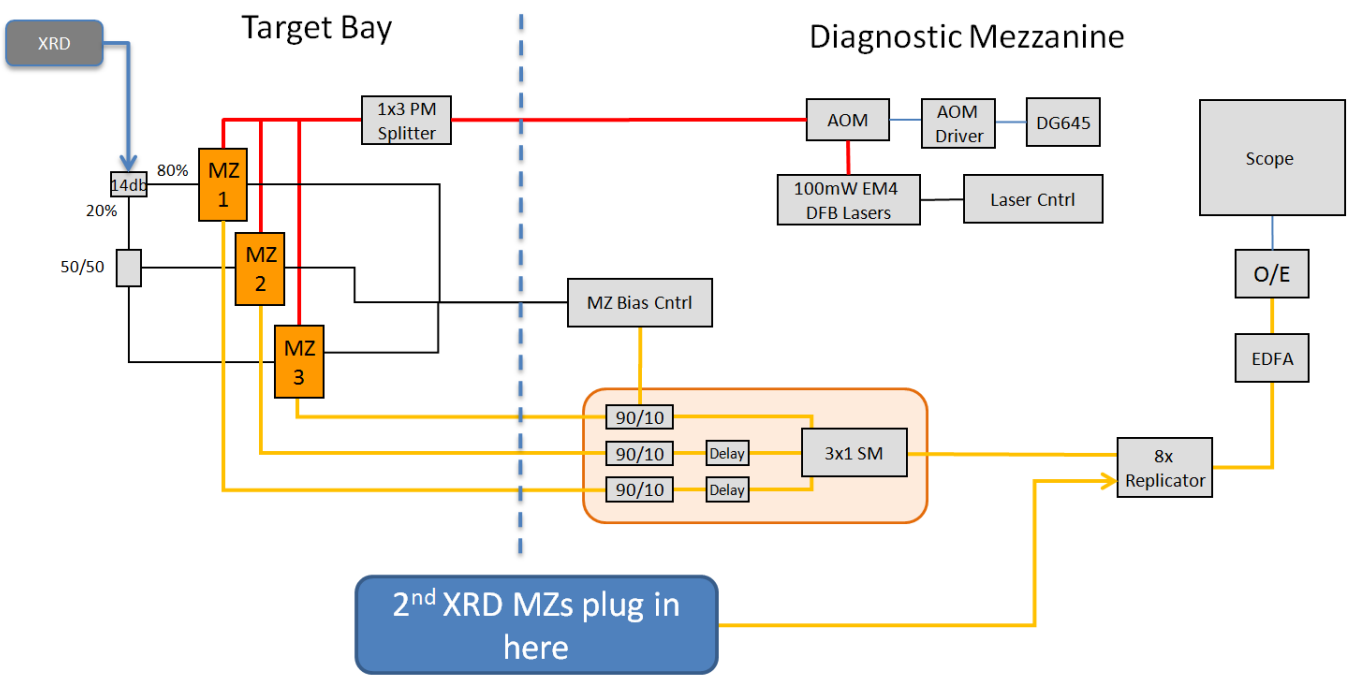

Figure 6. Dante Multiplexed EO System

Phase wrapping is an effective technique for reducing the noise on large signals $(>15 \mathrm{v})$ but does not help in the detection of $50 \mathrm{mV}$ signals. Low noise components, the use of optical vs. electrical amplification, custom bias control ${ }^{1}$, and optical replication ${ }^{2}$ are required.
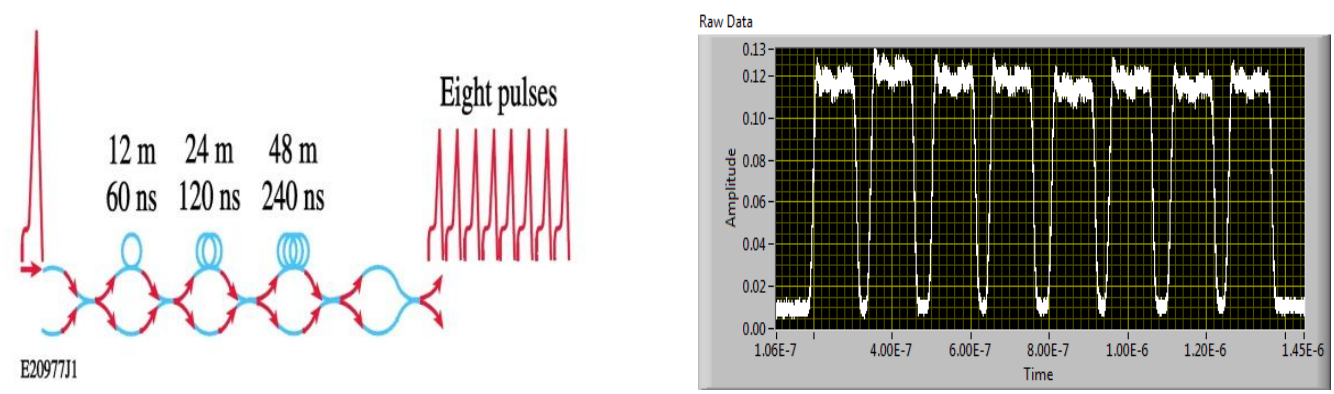

Figure 7. The replicator is constructed with $2 \times 2$ optical splitters. The outputs of the three stages are connected to the inputs of the subsequent stage with one of the connections having an additional length of fiber. Therefore, the eight replicated pulses arrive at the output at different times. This allows averaging of any noise source downstream of the replicator (i.e. digitizer noise). The trace shown was collected with fiber delays of approximately $150 \mathrm{~ns}, 300 \mathrm{~ns}$ and $600 \mathrm{~ns}$ in the replicator. 

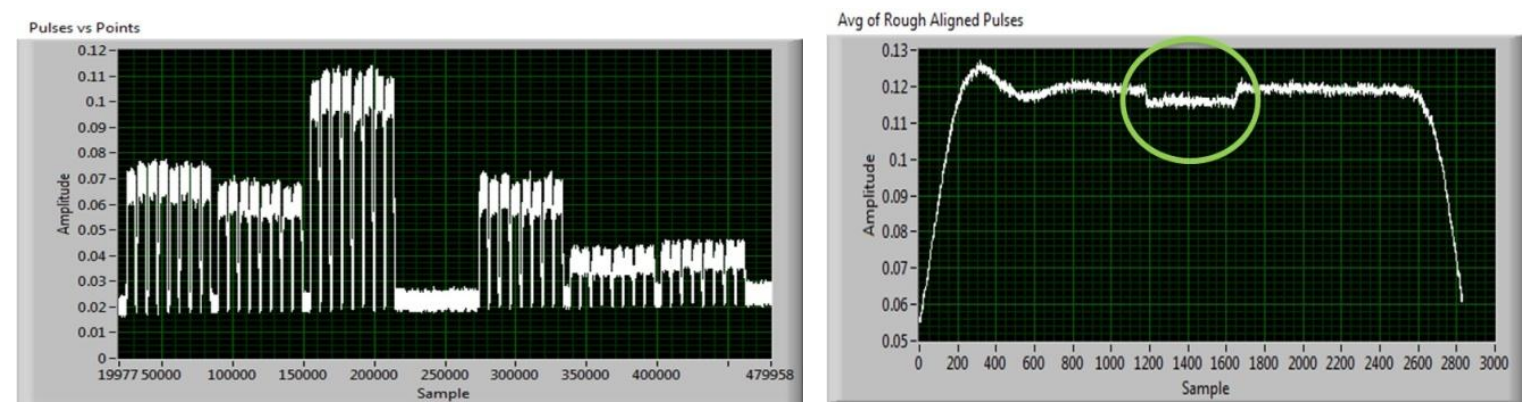

Figure 8. Mach-Zehnders fully multiplexed onto a single digitizer channel while $60 \mathrm{mV}$ input pulse clearly discernable in average of 8 replicated pulses

\section{Conclusion:}

Exploiting the phase wrapping characteristic of the Mach-Zehnders effectively increased the SNR beyond the limit of the recording digitizer for large signals in the SPBT configuration. Using the IRF derived at COMET and offline simulations, the Mach-Zehnder system can meet the SPBT specification of $<30$ ps Bang Time Error given an input signal $>15 \mathrm{v}$, with a Response time $<85 \mathrm{ps}$.

DANTE XRD multiplexing (6 Mach-Zehnders, 2 XRDs) has been demonstrated as has the $\sim 50 \mathrm{mv}$ signal detection capability of the DANTE EO system. We are awaiting the new bias controller which is expected to further increase the SNR adding margin to the detection system. Tests have also been performed with a $50 \mathrm{~V}$ input and previous experience leads confidence the 200v upper specification will also be satisfied shortly.

\section{REFERENCES}

${ }^{1}$ E. I. Moses, Fusion Sci. Technol. 54, 361 (2008).

${ }^{2}$ http://www.lecroy.com/labmaster/.

${ }^{3}$ E. L. Dewald et al., Rev. Sci. Instrum. 75, 3759 (2004).

${ }^{4}$ E. L. Wooten et al., IEEE J. Sel. Top. Quantum Electron. 6, 69 (2000).

${ }^{5}$ Generic Reliability Assurance Requirements for Optoelectronic Devices Used in Telecommunications Equipment, Telcordia Technologies, Inc., Piscataway, NJ, Document No. GR-468-CORE, Issue 2 (September 2004).

${ }^{6}$ Fiberdyne Labs, Inc., accessed 26 April 2012, http://www.fiberdyne. com/products/itu-grid.html.

${ }^{7}$ E. I. Ackerman and C. H. Cox III, in International Topical Meeting on Microwave Photonics, 2000 (IEEE, New York, 2000), p. 121.

${ }^{8}$ W. R. Donaldson, J. R. Marciante, and R. G. Roides, IEEE J. Quantum Electron. 46, 191 (2010).

${ }^{9}$ IEEE Std. 1241-2000, IEEE Standard for Terminology and Test Methods for Analog-to-Digital Converters (IEEE, New York, 2001).

2J. M. Mack, S. E. Caldwell, S. C. Evans, T. J. Sedillo, D. C. Wilson, C. S. Young, C. J. Horsfield, R. L. Griffith and R. A. Lerche, "Multiplexed Gas Cherenkov Detector for Reaction-History Measurements," Rev. Sci. Instrum., 77, 10E728.1 (2006).

3 H. W. Herrmann, et al., "Diagnosing ICF Gamma Ray Physics," Rev. Sci. Instrum., 81, 10D333 (2010). E. K. Miller, H. W. Herrmann, W. Stoeffl and C. J. Horsfield, "Mach-Zehnder Fiber-optic Links for Reaction History Measurements at the National Ignition Facility", Journal of Physics: Conference Series, 244, 032055 (2010)

This work performed under the auspices of the U.S. Department of Energy by Lawrence Livermore National Laboratory under Contract DE-AC52-07NA27344. 